\title{
Graphene Materials Strengthen Aqueous Polyurethane Adhesives
}

\author{
Luca Cristofolini, ${ }^{\dagger}$ Gloria Guidetti, ${ }^{\ddagger}$ Kavin Morellato, ${ }^{\dagger}$ Marco Gibertini, ${ }^{\S}$ Matteo Calvaresi, ${ }^{\ddagger}$ \\ Francesco Zerbetto, ${ }^{\ddagger}$ Marco Montalti, ${ }^{\ddagger}$ and Giuseppe Falini ${ }^{*} \neq \odot$ \\ ${ }^{\dagger}$ Department of Industrial Engineering (DIN), Alma Mater Studiorum-Università di Bologna, I-40126 Bologna, Italy \\ "Department of Chemistry "Giacomo Ciamician", Alma Mater Studiorum-Università di Bologna, Via Selmi 2, I-40126 Bologna, \\ Italy \\ ${ }^{\S}$ FacGB s.r.l., Via S. Pertini 36, I-62012 Civitanova Marche, Italy
}

Supporting Information

ABSTRACT: Carboxyl-functionalized graphene platelets (GP) and graphene oxide (GO) sheets were added to a commercial aqueous adhesive dispersion of thermoplastic polyurethane (TP) (Idrotex 200 from FacGB s.r.l.). For both additives, the weight percentage was of industrial interest, 0.01 and $0.1 \mathrm{wt} \%$. The addition of GP/GO was carried out in a simple and scalable-up process that can be applied to other materials and additives. Mechanical, peel tests were applied on polyurethane strips ( $75 \mathrm{~mm}$ long, $15 \mathrm{~mm}$ wide, and $1.5 \mathrm{~mm}$ thick) prepared cutting extruded sheets obtained using Estane 58091, a 70D aromatic polyester-based TP. The tests with $0.01 \mathrm{wt} \%$ of GP showed statistically significant higher forces at first failure and maximum forces with respect to the pristine adhesive. Sample characterization was carried out with scanning electron microscopy, infrared spectroscopy, X-ray diffraction, and thermal analysis. A mechanism is suggested for the improved performance of the low-dose GP adhesive.

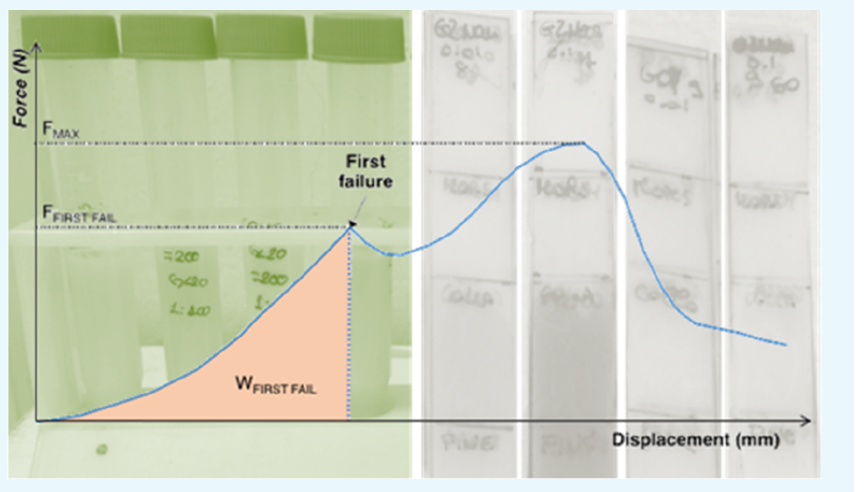

\section{INTRODUCTION}

In recent years, modern adhesives have delivered numerous advantages for constructors and processors alike. In particular, bonding materials quickly and securely is a reason why in many cases their use is considered a part of production processes. ${ }^{1}$

Synthetic thermoplastic adhesives are among the simplest forms of adhesives to handle. ${ }^{2}$ In particular, with respect to other types of materials, thermoplastic polyurethane (TP) adhesives have an excellent low-temperature resistance, together with good flexibility, toughness, and good wetting for a variety of substrates. An important advantage of these materials is the capability to tailor various products in order to meet different requirements for diverse industrial applications. ${ }^{3}$

The majority of the TP adhesive market products are based on aromatic isocyanates (e.g., methylene diphenyl diisocyanate). Aliphatic isocyanates are also finding increased interest because of their nonyelding light resistance. ${ }^{1}$

Essentially, these TP adhesives are high-concentration polymer solutions that can be spread on the surfaces to be bonded; the solvent slowly evaporates giving a solid polymer, which forms an effective bonded film. The surfaces are brought into contact before the solvent evaporates completely or after the polymer has been thermally reactivated.

Key features of TP adhesive films are the crystallization degree, the thermoplasticity, and the "open time" (time for bonding). These properties can be combined in various ways by changing the formulation of the TP starting material. In particular, the crystallization degree of TP films can be tuned over a wide range of values with a consequent increment of the hardness and the peel strength of adhesive. ${ }^{4}$

In order to influence the adhesive properties of TP films, several materials can be used as additives: organic molecules, ${ }^{5}$ nanoparticles (e.g., hydrophilic silica nanoparticles), ${ }^{6}$ and nanomaterials (e.g., graphene). ${ }^{7}$ For instance, adhesive nanocomposites of organically modified montmorillonite and polyurethane have been synthesized and their permeability to oxygen and water vapor has been measured. The water vapor permeation through the polyurethane nanocomposites was reduced more strongly than for oxygen, and a 50\% reduction was observed at 3 vol $\%$ silicate fraction. ${ }^{5}$

The addition of hydrophilic nanoparticles of silica favored the degree of phase separation between the hard (i.e., isocyanate + chain extender) and soft (i.e., polyol) segments of the TP adhesive. The tensile strength increased and the elongation at break of the TP adhesive decreased by increasing the silanol content of the silica nanoparticles. ${ }^{6}$

Additives such as graphene and graphene derivatives were used to enhance the mechanical and thermal properties of

Received: June 14, 2018

Accepted: July 26, 2018

Published: August 8, 2018 
polyurethane $\mathrm{e}^{7-11}$ or to provide electrical conductivity (EC) and anticorrosion properties of polyurethane coatings. ${ }^{12}$ Recent developments in the field of polyurethane/graphene nanocomposite showed interesting applications as shape memory, adsorbent, electromagnetic interference shielding, and gas barrier materials. ${ }^{13}$

Further improvements in EC were obtained with hybrid graphene/carbon nanotubes additives. At the same additive loading, the EC of hybrid filler systems was significantly higher than single filler systems $\left(0.77 \Omega^{-1} \mathrm{~m}^{-1}\right.$ at 5 wt $\%$ while the single filler system was not conductive). On the contrary, the best anticorrosion properties were obtained with low additive loading, which had better anticorrosion properties. ${ }^{12}$

Furthermore, a big issue that needs to be considered is the use of the solvent in adhesives. Recently, aqueous polyurethane dispersions have emerged as important alternatives to their solvent-based counterparts because of the increased awareness of health and environmental issues. ${ }^{14}$

The adhesive properties of TP dispersions showed a decrease in peel strength by increasing the molecular weight of polyethylenglycol. ${ }^{15}$ Moreover, solid content percentage, drying time, and storage stability suggested fast drying and greater stability of aqueous polyurethane dispersions. ${ }^{5}$

Choi et al. ${ }^{16}$ reported nanocomposites of aqueous TP dispersions reinforced with functionalized graphene sheets (up to $0.5 \mathrm{wt} \%)$. The preparation was carried out by casting a suspension of graphene sheets into an aqueous polyurethane dispersion. They observed that the addition of graphene brought some advantages in terms of enhanced EC and thermal resistance but also a decrement in tensile properties at high deformation (i.e., tensile strength and elongation at break). ${ }^{16}$

The aim of our study was to assess the improvement of the mechanical properties obtained by the addition of a small (and hence commercially relevant) amount of graphene to a commercial TP adhesive. More specifically, the following hypotheses were tested.

- Does the addition of graphene improve the first failure strength (defined as a drop of the force of $0.5 \mathrm{~N}$ ) of a commercial adhesive?

- Does it increase the mechanical energy absorbed before failure initiation?

- Does it increase its maximal strength?

More in detail, aqueous TP dispersions containing carboxylfunctionalized graphene platelets (GP) and graphene oxide (GO) sheets were prepared and their adhesive properties were measured. As the starting material, a commercial aqueous TP adhesive dispersion (Idrotex 200 from FacGB s.r.l.) was used. This aqueous TP adhesive dispersion was prepared according to the acetone process. ${ }^{17}$ The addition of the GP/GO additives was carried out having an industrial perspective, thus applying just one step mixing to the pristine adhesive.

\section{MATERIALS AND METHODS}

Materials. Idrotex 200 was kindly provided by Fac GB s.r.l. Idrotex 200 is a $52 \mathrm{wt} \%$ dispersion of an alkylic polyurethane anionomer (sulfinic) and silica particles (about $10 \mathrm{wt} \%$ ). It was prepared according to the so-called acetone process (ISBN 978-94-011-2924-4). The graphene suspension of GP and GO were obtained by using G2Nan and GO provided from Nanesa, Italy. The polyurethane strips $(75 \mathrm{~mm}$ long, $15 \mathrm{~mm}$ wide, and $1.5 \mathrm{~mm}$ thick) for mechanical tests were prepared cutting extruded sheets obtained using Estane 58091, a 70D aromatic polyester-based TP.

Composite TP Adhesive Preparation. Aqueous dispersions of GP or GO in ultrapure Milli-Q water were prepared by vigorous mixing of the starting materials in a concentration of $1 \mathrm{wt} \%$. Aliquots of GP or GO were added to the Idrotex 200. In a typical experiment, $20 \mathrm{~mL}$ of Idrotex 200 dispersion was poured in a $50 \mathrm{~mL}$ glass beaker and mechanically stirred using a $1 \mathrm{~cm}$ magnetic stir bar rotating at $1000 \mathrm{rpm}$. GP or GO was added to the dispersion during the stirring that ended after $30 \mathrm{~min}$. The mixing was carried out at room temperature. The composite dispersion was then bathsonicated for $2 \mathrm{~h}$ in an ice bath. Stability of the dispersion against centrifugation and aging was investigated in order to optimize the carbon material loading of the adhesive composition.

Samples prepared upon initial addition of 0.01 or $0.1 \mathrm{wt} \%$ of GP or GO (with respect to the aqueous Idrotex 200 dispersion) were named $\mathrm{GP}_{0.01 \%}, \mathrm{GP}_{0.1 \%}, \mathrm{GO}_{0.01 \%}$, and $\mathrm{GO}_{0.1 \%}$ (Table SI1). All samples showed good homogeneity. The gray colored suspensions, in fact, produced no precipitate upon centrifugation at $30 \mathrm{~g}$ for $5 \mathrm{~min}$ (Figure SI1), demonstrating that the carbon material had been completely, finely dispersed. Moreover, these suspensions, $\mathrm{GP}_{0.01 \%}, \mathrm{GP}_{0.1 \%}$, $\mathrm{GO}_{0.01 \%}$, and $\mathrm{GO}_{0.1 \%}$, having a final concentration of $\mathrm{GP} / \mathrm{GO}$ of either 0.01 or $0.1 \mathrm{wt} \%$, did not show formation of any precipitate for six months, demonstrating a very long-term shelf stability. On the contrary, suspensions prepared with a loading of GP/GO > 0.1 wt \% showed a tendency to stratify both during centrifugation and in aging experiments, demonstrating unsuitability for actual application. Mechanical properties will be analyzed only for $\mathrm{GP}_{0.01 \%}, \mathrm{GP}_{0.1 \%}, \mathrm{GO}_{0.01 \%}$, and $\mathrm{GO}_{0.1 \%}$. The characterization of the samples was carried on by spreading the different adhesive liquid dispersion on the surface of polyurethane strips, using about $200 \mu \mathrm{L} / \mathrm{cm}^{2}$. The strip dimensions were length $7.5 \mathrm{~cm}$, width $1.5 \mathrm{~cm}$, and thickness $1 \mathrm{~mm}$. They were glued for a length of $3.5 \mathrm{~cm}$. After solvent (water) evaporation, at room temperature and a relative humidity of less than $70 \%$, an adhesive film was formed with a thickness of about $150 \mu \mathrm{m}$. For mechanical peeling tests, a procedure adapted from the European Standard procedure EN 1392:2006:E. The adhesive films were heat-activated in a commercial oven at $80{ }^{\circ} \mathrm{C}$ for $15 \mathrm{~min}$. This was done because heat-activated adhesives (like Idrotex 200) do not form bonds until they are heated to a minimum activation temperature (typically $50-100^{\circ} \mathrm{C}$ ). Then, we bonded two strips by putting in contact the surfaces containing the reactivated adhesive for $10 \mathrm{~h}$ under a load of $10 \mathrm{~kg}$ (Figure SI2).

Methods. The optical microscopy observations of films were made with a Leica microscope equipped with a digital camera. The X-ray diffraction (XRD) patterns were collected using a PANalytical X'Pert Pro diffractometer, equipped with a copper anode and a fast $\mathrm{X}^{\prime}$ celerator counter $(\mathrm{K} \alpha$ radiation, $\lambda=$ $1.5418 \AA$ ); $2 \theta$ range from $5^{\circ}$ to $40^{\circ}$, step width $0.05^{\circ}$, and counting time $120 \mathrm{~s}$. Fourier-transform infrared spectra were collected in attenuated total reflection (ATR-FTIR) mode using a Thermo Scientific Nicolet iS10 FT-IR Spectrometer. Thermal gravimetrical analysis (TGA) of a small portion of the samples (3-5 mg) was carried out using a TA Instruments SDT 2960 , at a heating rate of $10{ }^{\circ} \mathrm{C} / \mathrm{min}$ from 30 to $600{ }^{\circ} \mathrm{C}$ in a nitrogen atmosphere (nitrogen flow rate of $100 \mathrm{~mL} / \mathrm{min}$ ). 
The scanning electron microscopy (SEM) observations were conducted using a Phenom microscope (FEI) on uncoated samples.

The electrical resistivity of each sample was measured using a four-point technique Jandel Multiheight Probe, an AMEL model 2053 potentiostat/galvanostat, and a Hewlett Packard 3478A multimeter.

Mechanical (peeling) tests were performed on polyurethane strips (length $7.5 \mathrm{~cm}$, width $1.5 \mathrm{~cm}$ ). Pairs of strips were glued for a length of $3.5 \mathrm{~cm}$ (Figures 1 and SI2). A typical T-peel test

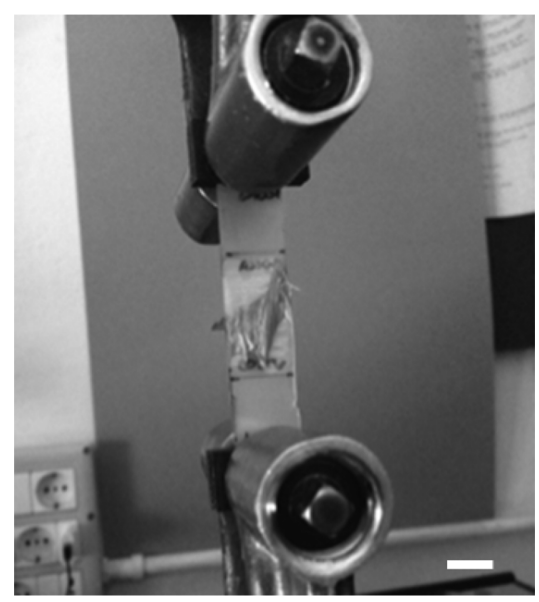

Figure 1. Photograph of a peeling test experiment. Scale bar: $1 \mathrm{~cm}$.

was carried out because the bonded substrates were flexible. The experiment was performed by anchoring the terminal 2 $\mathrm{cm}$ of the strips. With this geometry, $2 \mathrm{~cm}$ of strip remain between the clamp and the end of the bonded strips.

The specimens were allowed to season for at least 5 days at room temperature with a relative humidity of less than $70 \%$. The strength of bonding was measured at room temperature using a testing machine (model 4465, Instron Wolverhampton, UK) equipped with a $100 \mathrm{~N}$ load cell. A crosshead speed of 1 $\mathrm{mm} / \mathrm{s}$ was imposed, resulting in a test duration between 20 and 50 s. $N=8$ specimens were tested for each composition.

The results of mechanical peeling tests are reported in the force-displacement plots (Figure 2).

These data were examined to identify the following:

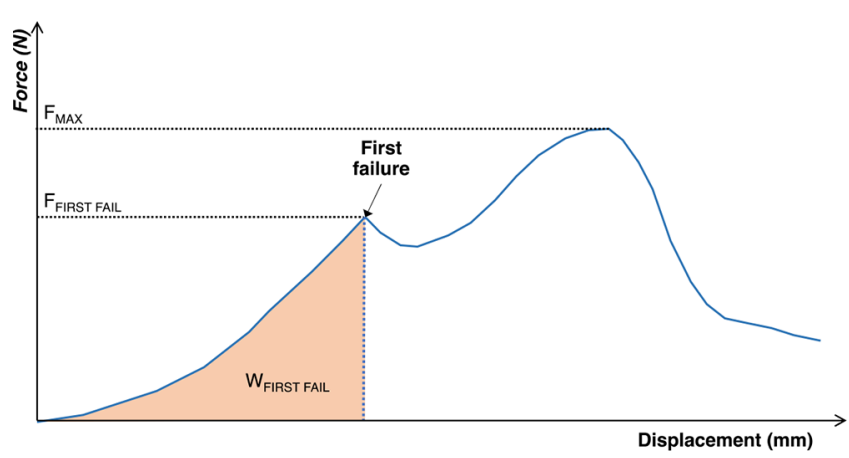

Figure 2. Typical force-displacement plot for the peel test. After identifying the point of failure initiation (defined as a drop of the force of $0.5 \mathrm{~N}$ ), the following parameters were computed: force to first failure $\left(F_{\text {FIRST FAIL }}\right)$, work to first failure $\left(W_{\text {FIRST FAIL }}\right)$, and maximal force $\left(F_{\mathrm{MAX}}\right)$. The figure does not report the scale on the axes as it shows a schematic profile. (i) The point of failure initiation (defined as a drop of the force of $0.5 \mathrm{~N})$;

(ii) The force (in newton) required to reach point of failure initiation together with the mechanical energy (work, in joule) to reach this point;

(iii) The maximum force reached in the test.

The work measured experimentally includes the work required to deform and peel the adhesives together with the work to deform (bending and stretch) the polyurethane strips. This component can affect the absolute value of the results but not their relative significance, which therefore is evaluated by a statistical treatment.

In order to analyze the significance of differences in variance between samples, the F-test was applied. As most differences were not statistically significant, parametric tests were applied. The significance of the different treatments was assessed with a one-way ANOVA (analysis of variance), with a least significant difference post-hoc. All statistical analyses were performed with StatPlus v6.0.3 for Mac (AnalystSoft, Walnut, CA, USA).

\section{RESULTS AND DISCUSSION}

Composite TP Adhesive Structural Characterization. The SEM images of composite TP adhesives films on polyurethane strips are shown in Figure 3.
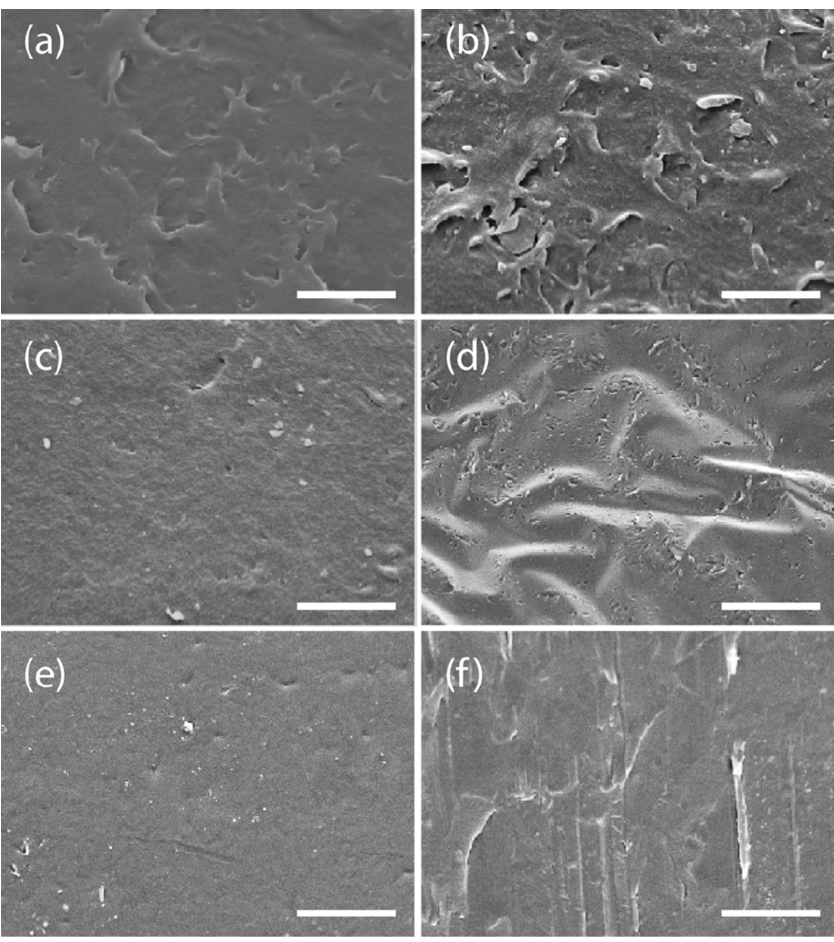

Figure 3. SEM images of films from composite aqueous TP adhesive containing (a) $\mathrm{GP}_{0.01 \%}$; (b) $\mathrm{GP}_{0.1 \%}$; (c) $\mathrm{GO}_{0.01 \%}$; (d) $\mathrm{GO}_{0.1 \%}$; (e) pristine; and (f) surface of the polyurethane strip. Scale bar: $5 \mu \mathrm{m}$.

In the absence of GP or GO, the adhesive displayed a very compact film that is morphologically similar to the surface of the polyurethane strip support, as can be seen comparing Figure 3e,f. The presence of GP or GO causes an increase of roughness of composite $\mathrm{TP}$ adhesive films, and this effect increases with the concentration of GP or GO from 0.01 to 0.1 wt \%, Figure $3 a-c$, compared to Figure $3 b-d$ and Figure $3 e$. SEM images also showed that a concentration of additive of 
$0.1 \mathrm{wt} \%$ lead to a reorganization of the polymeric material in fibrous structures that generated cavities and holes, Figure $3 b-$ $\mathrm{d}$. This textural change is more marked in the presence of GP than GO. In addition, the images of Figure 3 demonstrated that the presence of GP or GO additives favors the aggregation of the silica nanoparticles (contained in the adhesive formulation), which appear as bright particles. All these observations could be justified considering a change in the drying mechanism of the adhesive induced by the presence of GP or GO, to which it could be associated a structural reorganization of the TP fibers and a phase separation. To evaluate if these changes in the textural organization were associated to a structural reorganization of the polyurethane fibers, the composite adhesive films were also investigated by ATR-FTIR spectroscopy and XRD. In Figure 4 the ATRFTIR spectra were reported.

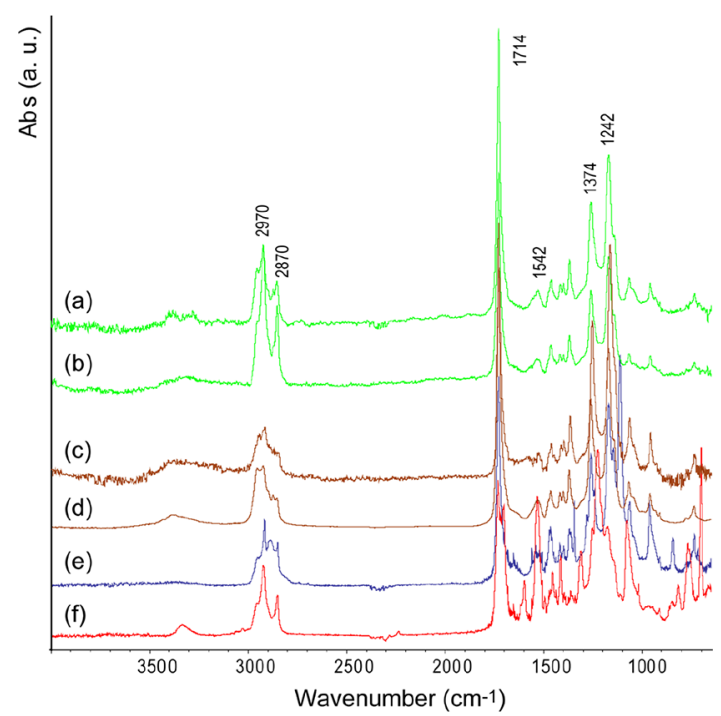

Figure 4. ATR-FTIR spectra of films from aqueous composite TP adhesive containing (a) $\mathrm{GP}_{0.01 \%}$; (b) $\mathrm{GP}_{0.1 \%}$; (c) $\mathrm{GO}_{0.01 \%}$; (d) $\mathrm{GO}_{0.1 \%}$; (e) pristine; and (f) surface of the polyurethane strip.

They showed the typical absorption bands corresponding to the hard segments and to soft segments groups of polyurethanes. $^{18}$ The main ATR-FTIR absorption bands are reported and assigned in Table SI2. No band shifts was observed, and only small differences in the relative intensity of absorption bands in the region 3000-2800 and 1200-900 $\mathrm{cm}^{-1}$ were detected. These changes could be associated to a minimal reorganization of soft segments, also considering the observations of fibers by SEM only in the presence of $\mathrm{GP}_{0.1 \%}$, or $\mathrm{GO}_{0.1 \%}$. However, the absence of the ATR-FTIR band shift suggests that the interactions among the functional groups of the TP have not been altered by the addition of GP or GO. This observation on the unaffected TP structural organization was further supported by the analysis of the XRD patterns of the adhesive samples containing $\mathrm{GP}_{0.01 \%}, \mathrm{GP}_{0.1 \%}, \mathrm{GO}_{0.01 \%}$, or $\mathrm{GO}_{0.1 \%}$. They were very similar to the one of the reference TP adhesive, which was GP/GO-free. These patterns showed a broad reflection centered around $2 \theta=20^{\circ}$, suggesting that the starting material is mainly in an amorphous state, and several sharp diffraction peaks (Figure SI3) that have been indexed according to the diffraction of cristobalite. ${ }^{19}$ Differently, the thermal properties of the adhesives changed when GP or GO was present, as shown by the analyses of the TGA profiles of the composite adhesives and of the pristine one (Figure 5).

The main weight loss event, which for the pristine adhesive and the $\mathrm{GP}_{0.01 \%}$ or $\mathrm{GO}_{0.01 \%}$ occurs at about $370{ }^{\circ} \mathrm{C}$, is increased at about $398{ }^{\circ} \mathrm{C}$ in the presence of $\mathrm{GP}_{0.1 \%}$ or $\mathrm{GO}_{0.1 \%}$. That endothermic event has an enthalpy around $550 \mathrm{~J} / \mathrm{g}$ for the pristine material and the $\mathrm{GP}_{0.01 \%}$ or $\mathrm{GO}_{0.01 \%}$, which decreases to about 450 or $490 \mathrm{~J} / \mathrm{g}$ in the presence of $\mathrm{GP}_{0.1 \%}$ or $\mathrm{GO}_{0.1 \%}$, respectively.

This result may indicate that at the lower concentration GP or GO disperse among TP fibers without affecting their organization, the enthalpy of pyrolysis and the temperature of pyrolysis do not change, in agreement with SEM observations and ATR-FTIR and XRD investigations. On the contrary, when a high concentration of GP or GO is present, the main effect of GP and GO appears to be on the microfibrillar organization of $\mathrm{TP}$, with a reduction of the enthalpy of pyrolysis and an increase of the temperature of pyrolysis, in accord with the SEM observations.

The samples did not show any conductivity, which indicates that the low concentrations of GP and GO present in the composite TP adhesive do not result in percolation conductivity.

Composite TP Adhesive Mechanical Characterization. All the specimens exhibited a similar trend during the peel test, with a monotonic trend up to the initiation of failure, followed by a marked drop and a subsequent new increase of the force (Figure 2).

The composition had a significant effect both on the force to

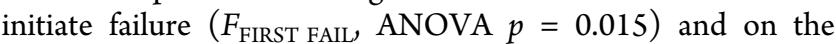
maximal force $\left(F_{\mathrm{MX}}\right.$, ANOVA $\left.p=0.019\right)$. However, there was no influence of the composition on the work to initiate failure $\left(W_{\text {FIRST FAIL }}\right.$ ANOVA $\left.p=0.054\right)$.

Figure 6 presents barcharts with $p$-values that show the following:

- The force to initiate failure $\left(F_{\text {FIRST FAIL }}\right)$ was significantly higher for both percentages of GP, compared to the pristine adhesive (respectively 1.65 and 1.87 times). Remarkably, the lower $0.01 \%$ of GP was also significantly stronger than the higher $0.1 \%$. Conversely, addition of GO caused a nonsignificant variation of $F_{\text {FIRST FAIL, with a slight increase and a slight decrease for }}$ the 0.1 and $0.01 \%$ additions, compared to the pristine adhesive. The $\mathrm{GP}_{0.01 \%}$ was also significantly stronger $\mathrm{GO}_{0.01 \%}$. The interspecimen variability for the $F_{\text {FIRST FAIL }}$ was similar (F-test, $p>0.05$ ) for all the compositions both in terms of standard deviation and as a coefficient of variation (ratio between standard deviation and mean value).

- When the maximum force $\left(F_{\mathrm{MAX}}\right)$ was considered, addition of both percentages of GP significantly increased the strength, compared to the pristine adhesive (respectively 1.36 and 1.76 times). Remarkably, the lower $0.01 \%$ of GP was again stronger than the higher $0.1 \%$ (but this difference was not statistically significant). Addition of GO caused a slight increase and a slight decrease of $F_{\mathrm{MAX}}$ for $\mathrm{GO}_{0.1 \%}$ and $\mathrm{GO}_{0.01 \%}$, compared to the pristine adhesive (this effect was not statistically significant). The $\mathrm{GP}_{0.01 \%}$ was also significantly stronger $\mathrm{GO}_{0.01 \%}$. The interspecimen variability for the $F_{\mathrm{MAX}}$ was slightly larger for $\mathrm{GP}_{0.01 \%}$ and $\mathrm{GO}_{0.1 \%}$ than for the pristine adhesive (F-test, $p=0.029$ and 0.015 

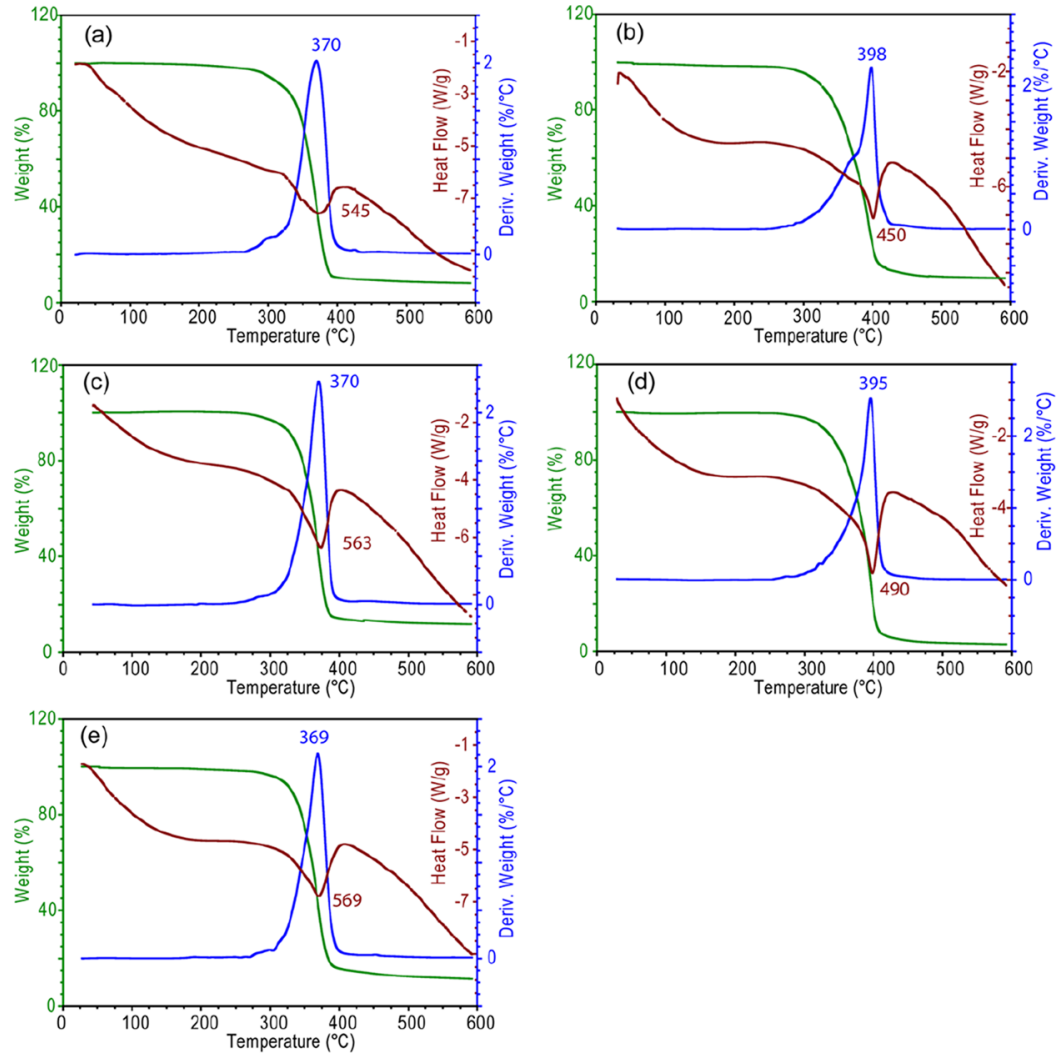

Figure 5. Thermal analyses of the composite TP adhesive containing

(a) $\mathrm{GP}_{0.01 \%}$; (b) $\mathrm{GP}_{0.1 \%}$; (c) $\mathrm{GO}_{0.01 \%}$; (d) $\mathrm{GO}_{0.1 \%}$; and (e) pristine.

respectively). Similar interspecimen variability was observed for all other compositions (F-test, $p>0.05)$.

- The largest work to first failure $\left(W_{\text {FIRST FAIL }}\right)$ was found with a $\mathrm{GP}_{0.01 \%}$ : this was larger than for the pristine adhesive, and for $\mathrm{GP}_{0.1 \%}$. The work for $\mathrm{GO}_{0.1 \%}$ was slightly larger than the pristine adhesive, whereas $\mathrm{GO}_{0.01 \%}$ required the minimum amount of energy. All such differences for $W_{\text {FIRST FAIL }}$ were not statistically significant. The interspecimen variability for the $W_{\text {FIRST FAIL }}$ was similar for all the compositions both in terms of standard deviation and as a coefficient of variation (ratio between standard deviation and mean value), the $\mathrm{GP}_{0.1 \%}$ and $\mathrm{GP}_{0.01 \%}$ being slightly more repeatable than all the others (F-test, $p>0.05)$.

The rationale for the different behavior in the presence of GP and GO can be addressed considering their diverse chemical structure and capability to affect the polyurethane fiber organization. In the case of GP, the strength increases for the 0.01 wt $\%$ composite. As the mass fraction is increased further, the strength falls to lower values. This behavior is typical for composites and is usually explained by aggregation effects. ${ }^{20-22}$ Almost identical behavior was recently observed for composites of polyurethane reinforced with functionalized nanotubes. ${ }^{23}$ The reduction in strength was explained in terms of interactions between the nanotubes and the polyurethane soft segments, resulting in failure at lower stress. In the case of $\mathrm{GO}$, on the contrary, the oxygen containing groups have been reported to interact with the carbonyl group from the hard segment of the polymer chain causing a disruption of hydrogen bonding of polyurethanes. ${ }^{24}$ This effect dominates at $0.01 \%$ loading causing a decrease of the mechanical performances.
The beneficial effect of GP on the mechanical properties of the adhesive finds justification from the thermal, spectroscopic, and morphological observations, all suggesting an incorporation GP within the TP adhesive. This has to increase the chemical compatibility between the composite TP adhesive and adherent surfaces, probably due to the cumulative effects of intermolecular interactions between the GP and TP networks, leading to a significant increase of the force to reach first failure and the maximum force in comparison with the corresponding joints with unfilled adherents.

In addition, the data reported in Figure 6 indicate that the increase of the concentration of GP and GO by a factor of 10 (from 0.01 to $0.1 \mathrm{wt} \%$ ) causes a decrease of the force to the first failure and of the maximum force. This is likely due to the agglomeration of GP and GO causing heterogeneity in large domains at the interface between adherents and composite TP adhesives. Accordingly, the SEM observations show that the higher concentration of GP and GO makes inhomogeneity (presence of cavities) in the composite TP adhesive film coverage. The influence of aggregation effect of graphene on the mechanical properties of nanocomposite polymers has been investigated. ${ }^{25,26}$ Interfacial interactions between polymers and graphene-based materials play a key role in the mechanical integrity of the corresponding nanocomposite and their mechanical performance. ${ }^{27} \mathrm{~A}$ fine control of dispersion and distribution of the graphene nanofillers ensures the optimal exposure of the graphene surface to the polymer matrix and an effective reinforcement of the mechanical properties. $^{25}$ Once dispersed, the graphene large surface tovolume ratio might result in high binding efficiency, but strong interfacial binding can alter the macromolecular conformation in the vicinity of the filler surface. ${ }^{28,29}$ Thus, the addition of an appropriate amount of graphene into an adhesive formulation 

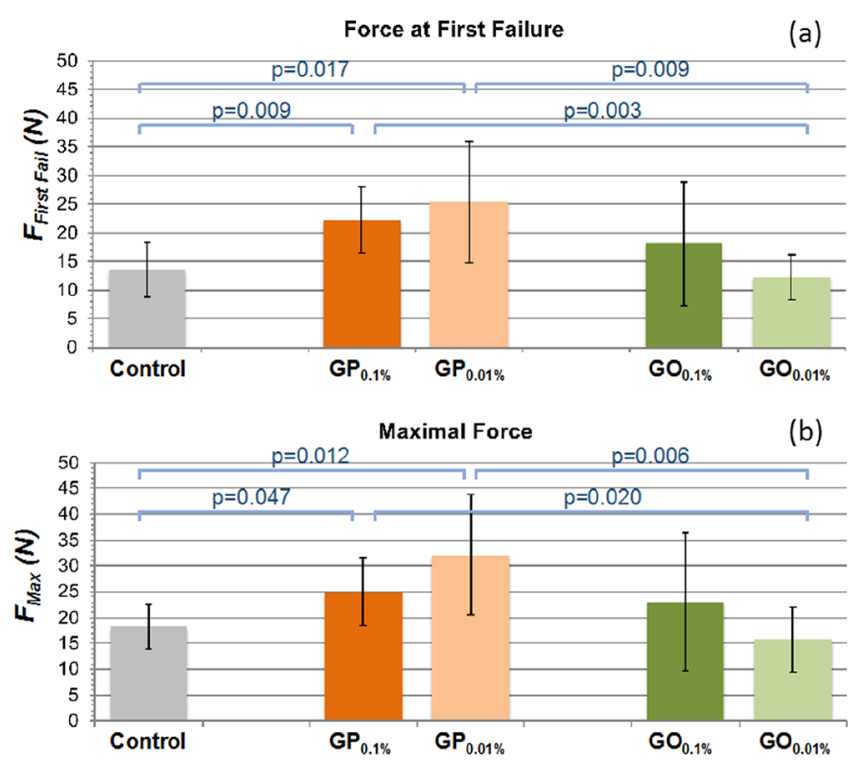

Work to First Failure

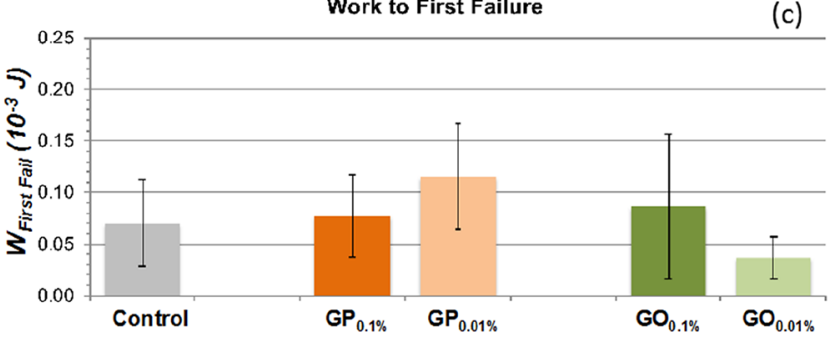

Figure 6. Comparison of the mechanical properties for the different compositions: $(a)$ force to reach the first failure $\left(F_{\text {FIRST FAIL }}\right)$; $(b)$ maximum force throughout the test $\left(F_{\mathrm{MAX}}\right)$; and $(\mathrm{c})$ work to reach the first failure ( $\left.W_{\text {FIRST FAIL }}\right)$. The one-way ANOVA indicated an overall significance of the effect of the composition on the force to first failure and maximal force, but not for the work to first failure. Consequently, the significance of differences between pairs is reported where the post-hoc test indicated a significant difference; the $p$-values are not indicated when $p>0.1$.

causes a significant improvement in the mechanical performances. The reinforcement effect generated by the graphene is due to the improvement of the attractive interactions between the adhesive matrix and the dispersed graphene sheets. On the opposite, the addition of an excessive amount of graphene is deleterious for the mechanical properties because graphene sheets start to aggregate into the matrix, forming heterogeneous domains that degrade the mechanical properties of the composite.

Overall, the above observations indicate a favorable effect of low concentrations of GP, and also GO, on some mechanical performances of the commercial Idrotec 200 adhesive.

These data are in line with previous studies showing that the peculiar properties of GP and GO sheets, among which there is high mechanical resistance, electric conduction, and high surface area, have been exploited in many industrial areas. In this case, the GP peculiarities are used to improve two mechanical properties of composite TP adhesive, of crucial importance for several applications (e.g., textile and footwear industry).

GP and GO have been already used as additives in epoxy-, ${ }^{20,30}$ polyvinyl acetate-, ${ }^{31}$ and polyurethane ${ }^{16}$-based adhesives. Epoxy adhesive based on tetraglycidylmethylene dianiline filled with graphene at a concentration of $1 \mathrm{wt} \%$ significantly enhanced the mechanical behavior of the bonded joints. The inclusion of $4 \mathrm{wt} \%$ graphene did not have a significant effect on the mechanical performance. ${ }^{20}$ While polyvinyl acetate containing of $0.1 \mathrm{vol} \%$ of exfoliated graphene increased $50 \%$ in stiffness and a $100 \%$ in tensile strength. ${ }^{31}$

It is important to emphasize that GP and GO have been added to commercial aqueous TP dispersions (Idrotex 200, FacGB s.r.l.). The synthesis of the composite TP adhesives is simple, based on commercially available materials and easy to scale-up. All the processes are carried out in water dispersions, making them environmentally attractive and suitable to the principles of the green chemistry. Furthermore, the low concentrations of GP and GO additives used in the samples make the final product economically interesting.

\section{CONCLUSIONS}

This research fits well in the context of new aqueous adhesives based on polyurethane, which having a growing commercial interest $^{32}$ is a theme that gathers attention both for basic and applied science. The experimental data show that the addition of a concentration of GP as low as 0.01 wt \% to Idrotex 200 allows to obtain a significant increase of first failure and maximum force in mechanical peeling tests. Interestingly, a less marked effect was obtained using GO or increasing the concentration of GP/GO to 0.1 wt $\%$. The addition of GP/GO is a simple and scalable-up process that can be applied to other materials and additives.

\section{ASSOCIATED CONTENT}

\section{S Supporting Information}

The Supporting Information is available free of charge on the ACS Publications website at DOI: 10.1021/acsomega.8b01342.

Pictures of dispersions of aqueous composite PU adhesive and polyurethane strips glued with aqueous composite TP adhesives containing GP/GO; X-ray diffraction patterns of films from composite aqueous PU adhesive containing GP/GO; composition of the GP- and GO-doped adhesives; and assignment for some of the observed ATR FTIR bands (PDF)

\section{AUTHOR INFORMATION}

\section{Corresponding Author}

*E-mail: giuseppe.falini@unibo.it.

ORCID

Matteo Calvaresi: 0000-0002-9583-2146

Francesco Zerbetto: 0000-0002-2419-057X

Giuseppe Falini: 0000-0002-2367-3721

Notes

The authors declare no competing financial interest.

\section{ACKNOWLEDGMENTS}

This project has received funding from the European Union's Horizon 2020 research and innovation programme under grant agreement no. GrapheneCore2 785219 (Graphene Flagship).

\section{REFERENCES}

(1) Bart, J. C. J. Additives in Polymers: Industrial Analysis and Applications; John Wiley \& Sons: Hoboken, NJ, USA, 2005. 
(2) Bartz, K. W.; Higgins, J. J.; Berejka, A. J.; Di Cresce, A. J. Thermoplastic Adhesive Compositions. U.S. Patent 3,868,433 A, Feb 25, 1975.

(3) Lee, S. Thermoplastic Polyurethane Markets in the EU: Production, Technology, Applications and Trends; Rapra Technology Limited, 1998.

(4) Lin, Y. G.; Mallin, D. T.; Chien, J. C. W.; Winter, H. H. Dynamic mechanical measurement of crystallization-induced gelation in thermoplastic elastomeric poly(propylene). Macromolecules 1991, $24,850-854$.

(5) Osman, M. A.; Mittal, V.; Morbidelli, M.; Suter, U. W. Polyurethane Adhesive Nanocomposites as Gas Permeation Barrier. Macromolecules 2003, 36, 9851-9858.

(6) Vega-Baudrit, J.; Navarro-Bañón, V.; Vázquez, P.; MartínMartínez, J. M. Addition of Nanosilicas with Different Silanol Content to Thermoplastic Polyurethane Adhesives. Int. J. Adhesion Adhes. 2006, 26, 378-387.

(7) Strankowski, M.; Korzeniewski, P.; Strankowska, J.; Anu, A. S.; Thomas, S. Morphology, Mechanical and Thermal Properties of Thermoplastic Polyurethane Containing Reduced Graphene Oxide and Graphene Nanoplatelets. Materials 2018, 11, 82.

(8) Chen, K.; Tian, Q.; Tian, C.; Yan, G.; Cao, F.; Liang, S.; Wang, $X$. Mechanical Reinforcement in Thermoplastic Polyurethane Nanocomposite Incorporated with Polydopamine Functionalized Graphene Nanoplatelet. Ind. Eng. Chem. Res. 2017, 56, 11827-11838.

(9) Scognamillo, S.; Gioffredi, E.; Piccinini, M.; Lazzari, M.; Alzari, V.; Nuvoli, D.; Sanna, R.; Piga, D.; Malucelli, G.; Mariani, A. Synthesis and Characterization of Nanocomposites of Thermoplastic Polyurethane with both Graphene and Graphene Nanoribbon Fillers. Polymer 2012, 53, 4019-4024.

(10) Bera, M.; Maji, P. K. Effect of structural disparity of graphenebased materials on thermo-mechanical and surface properties of thermoplastic polyurethane nanocomposites. Polymer 2017, 119, $118-133$.

(11) Ren, H.; Zhou, Y.; He, M.; Xu, R.; Ding, B.; Zhong, X.; Tong, Y.; Fan, L.; Cai, Z.; Shen, H.; Huang, Y. Enhanced Mechanical Properties of Silica Nanoparticle-Covered Cross-Linking Graphene Oxide Filled Thermoplastic Polyurethane Composite. New J. Chem. 2018, 42, 3069-3077.

(12) Tong, Y.; Bohm, S.; Song, M. The Capability of Graphene on Improving the Electrical Conductivity and Anti-Corrosion Properties of Polyurethane Coatings. Appl. Surf. Sci. 2017, 424, 72-81.

(13) Kausar, A. Emerging Research Trends in Polyurethane/ Graphene Nanocomposite: A Review. Polym. Plast. Technol. Eng. 2017, 56, 1468-1486.

(14) Kim, B. K. Aqueous Polyurethane Dispersions. Colloid Polym. Sci. 1996, 274, 599-611.

(15) Mumtaz, F.; Zuber, M.; Zia, K. M.; Jamil, T.; Hussain, R. Synthesis and Properties of Aqueous Polyurethane Dispersions: Influence of Molecular Weight of Polyethylene Glycol. Korean J. Chem. Eng. 2013, 30, 2259-2263.

(16) Choi, S. H.; Kim, D. H.; Raghu, A. V.; Reddy, K. R.; Lee, H.-I.; Yoon, K. S.; Jeong, H. M.; Kim, B. K. Properties of Graphene/ Waterborne Polyurethane Nanocomposites Cast from Colloidal Dispersion Mixtures. J. Macromol. Sci., Part B: Phys. 2012, 51, 197207.

(17) Hepburn, C. Polyurethane Elastomer, 2nd ed.; Elsevier: London, New York, 1992.

(18) Lee, H. S.; Wang, Y. K.; Hsu, S. L. Spectroscopic Analysis of Phase Separation Behavior of Model Polyurethanes. Macromolecules 1987, 20, 2089-2095.

(19) Pluth, J. J.; Smith, J. V.; Faber, J. Crystal Structure of Low Cristobalite at 10, 293, and $473 \mathrm{~K}$ : Variation of Framework Geometry with Temperature. J. Appl. Phys. 1985, 57, 1045-1049.

(20) Guadagno, L.; Sarno, M.; Vietri, U.; Raimondo, M.; Cirillo, C.; Ciambelli, P. Graphene-Based Structural Adhesive to Enhance Adhesion Performance. RSC Adv. 2015, 5, 27874-27886.

(21) Khan, U.; May, P.; O’Neill, A.; Coleman, J. N. Development of Stiff, Strong, yet Tough Composites by the Addition of Solvent Exfoliated Graphene to Polyurethane. Carbon 2010, 48, 4035-4041.
(22) Coleman, J. N.; Khan, U.; Blau, W. J.; Gun'ko, Y. K. Small but strong: A review of the mechanical properties of carbon nanotubepolymer composites. Carbon 2006, 44, 1624-1652.

(23) Khan, U.; Blighe, F. M.; Coleman, J. N. Selective Mechanical Reinforcement of Thermoplastic Polyurethane by Targeted Insertion of Functionalized SWCNTs. J. Phys. Chem. C 2010, 114, 1140111408.

(24) Liu, S.; Tian, M.; Yan, B.; Yao, Y.; Zhang, L.; Nishi, T.; Ning, N. High Performance Dielectric Elastomers by Partially Reduced Graphene Oxide and Disruption of Hydrogen Bonding of Polyurethanes. Polymer 2015, 56, 375-384.

(25) Hu, K.; Kulkarni, D. D.; Choi, I.; Tsukruk, V. V. Graphenepolymer nanocomposites for structural and functional applications. Prog. Polym. Sci. 2014, 39, 1934-1972.

(26) Kim, H.; Abdala, A. A.; Macosko, C. W. Graphene/Polymer Nanocomposites. Macromolecules 2010, 43, 6515-6530.

(27) Terrones, M.; Martín, O.; González, M.; Pozuelo, J.; Serrano, B.; Cabanelas, J. C.; Vega-Díaz, S. M.; Baselga, J. Interphases in Graphene Polymer-based Nanocomposites: Achievements and Challenges. Adv. Mater. 2011, 23, 5302-5310.

(28) Lipatov, Y. S. Interfaces in polymer-polymer composites. In Controlled Interphases in Composite Materials; Ishida, H., Ed.; Elsevier Science Publ Co. Inc.: Amsterdam, 1990; pp 599-611.

(29) Fang, M.; Wang, K.; Lu, H.; Yang, Y.; Nutt, S. Covalent polymer functionalization of graphene nanosheets and mechanical properties of composites. J. Mater. Chem. 2009, 19, 7098-7105.

(30) Marami, G.; Nazari, S. A.; Faghidian, S. A.; Vakili-Tahami, F.; Etemadi, S. Improving the Mechanical Behavior of the Adhesively Bonded Joints Using RGO Additive. Int. J. Adhesion Adhes. 2016, 70, 277-286.

(31) Khan, U.; May, P.; Porwal, H.; Nawaz, K.; Coleman, J. N. Improved Adhesive Strength and Toughness of Polyvinyl Acetate Glue on Addition of Small Quantities of Graphene. ACS Appl. Mater. Interfaces 2013, 5, 1423-1428.

(32) Meier-Westhues, U. Polyurethanes: Coatings, Adhesives and Sealants; Vincentz Network GmbH \& Co KG: Hannover, 2007. 